\title{
MODELING VISCOELASTIC FLOWS USING REFLECTED STOCHASTIC DIFFERENTIAL EQUATIONS*
}

\author{
A. BONITO ${ }^{\dagger}$, A. LOZINSKI ${ }^{\ddagger}$, AND T. MOUNTFORD ${ }^{\S}$
}

\begin{abstract}
A reflected diffusion process is proposed for modeling of viscoelastic fluids. In order to define the viscoelastic stress tensor, the reflected diffusion process is approximated by Itô diffusions with a penalization factor in the drift term. Convergence of the stress tensor approximation is proved and an expression for the limiting stress tensor in terms of the reflected process itself is provided.
\end{abstract}

Key words. Viscoelastic flows, dumbbell models, reflected stochastic differential equations.

AMS subject classifications. 60H10, 60H35, 76A05.

\section{Introduction}

In this article, we are concerned with a question related to the modeling of nonNewtonian fluids. In general, the modeling of fluid motion is based on a relation between the velocity field and the Cauchy stress tensor $T$. The simplest example of such a relation is the Newtonian one, $T=\eta\left(\nabla u+(\nabla u)^{T}\right)-p I$, where $\eta, u, p$ are the viscosity, velocity, and pressure in the fluid and.$^{T}$ denotes the matrix transposition operator. However, some fluids with complex internal structure may require more complicated relations, in which the stress may depend on the history of the velocity. More specifically, we shall consider here polymer solutions in which the contribution of interactions inside the long polymer molecules and their bombarding by the surrounding molecules should be taken into account. The simplest modeling of such a fluid consists in representing the polymeric molecules by "dumbbells", i.e. two beads connected by a spring and subject to random Brownian forces; refer to [2]. The distribution for the orientation and extension of the dumbbells is given by a stochastic process $X_{t}$ representing the vector connecting the two beads of the dumbbell. In the case of dilute solutions, when the interactions between the molecules of the polymer can be disregarded, the evolution of $X_{t}$ is governed by the following stochastic differential equation (SDE) (see [10]),

$$
d X_{t}=\left(\kappa(t) X_{t}-\frac{1}{2 \lambda} F\left(X_{t}\right)\right) d t+\sqrt{\frac{1}{\lambda}} d B_{t}
$$

where $\kappa$ is the velocity gradient tensor $\left(\kappa_{i j}=\partial u_{i} / \partial x_{j}\right), \lambda$ is the relaxation time of the fluid, and $B_{t}$ is the Brownian motion. $F($.$) in (1.1) is the the force of the spring$ connecting the beads. The Cauchy stress tensor $T$ is the sum of a Newtonian contribution $\eta_{s}\left(\nabla \mathbf{u}+\nabla \mathbf{u}^{T}\right)-p I$ with the solvent viscosity $\eta_{s}$ and the polymeric contribution $\tau$ known as the extra-stress tensor that can be computed by the Kramers expression (see $[2$, p. 69 , vol. II])

$$
\tau(t)=\frac{\eta_{p}}{\lambda}\left(-I+\mathbb{E}\left(X_{t} \otimes F\left(X_{t}\right)\right)\right),
$$

\footnotetext{
*Recieved: September 25, 2008; accpeted (in revised version): May 14, 2009.

$\dagger$ Department of Mathematics, Texas A\&M University, 3368 TAMU, College Station, TX 778433368, USA (bonito@math.tamu.edu).

Partially supported by NSF grant DMS-0914977 and SNSF fellowship PBEL2-114311.

¥Université Paul Sabatier, Institut de Mathématiques de Toulouse, 118 route de Narbonne, F31062 Toulouse Cedex 9, France (alexei.lozinski@math.univ-toulouse.fr).

§Section de mathématiques, Ecole Polytechnique Fédérale de Lausanne, CH-1015 Lausanne, Switzerland (thomas.mountford@epfl.ch).
} 
where the symbol $\otimes$ denotes the tensor product of two vectors and $\mathbb{E}(\cdot)$ is the expectation. The parameter $\eta_{p}$ is referred to as the polymeric viscosity.

The choice of the function $F($.$) should be done in principle by a more detailed$ description of intermolecular forces in the polymer. One usually starts from representing the polymer by a chain consisting of a great number of links, each of a fixed length, which can be either completely independent of each other (Freely Jointed Chain) or have random directions while keeping a fixed angle between the adjacent links (Freely Rotating Chain). In both cases, the random vector $X_{t}$ in (1.1) should represent approximately the end-to-end vector of the long chain, and one can show by considering the equilibrium distribution and applying the Central Limit Theorem that the force law should be linear $F(X)=X$ for small extensions $X$. However, such a law is certainly unrealistic when the chain is almost completely extended as it allows the dumbbells to be extended infinitely. A mathematically attractive option ensuring the finite extensibility is to introduce the force as the subdifferential of the potential

$$
\Pi(X)=\left\{\begin{array}{l}
\frac{1}{2}|X|^{2}, \text { if }|X|<R \\
+\infty, \text { otherwise }
\end{array}\right.
$$

for some fixed $R$. It means physically that the force $F($.$) is supposed to be linear$ $F(X)=X$ for the extensions $|X|<R$, but when $|X|=R$ the magnitude of the force becomes such that it prohibits the dumbbell to extend further. The SDE with the drift being a subdifferential of an infinite potential is well known in the mathematical literature as the Skorohod problem; see for instance [1]. The SDE (1.1) with the force derived from (1.3) can be thus written as

$$
d X_{t}=\left(\kappa(t) X_{t}-\frac{1}{2 \lambda} X_{t}\right) d t-d \mu_{t}+\sqrt{\frac{1}{\lambda}} d B_{t}
$$

where $\mu_{t}$ is an unknown stochastic process imposing to the process to remain in the ball $\overline{B(0, R)}$. A precise definition in the mathematical viewpoint will be provided in the next section.

The interpretation of the stress tensor (1.2) is now not straight forward since the force is no longer given as a function of $X$. We therefore propose to approximate the force $\partial \Pi$ by a penalized one

$$
F^{\varepsilon}(X)=X+\frac{1}{\varepsilon} \beta(X)
$$

where $\beta(X)=X-\pi(X)$ and $\pi(X)$ is the projection of $X$ on $\overline{B(0, R)}$, i.e.,

$$
\pi(X)=\left\{\begin{array}{l}
X, \text { if }|X| \leq R \\
\frac{R}{|X|} X, \text { otherwise }
\end{array}\right.
$$

The solution of (1.4) is thus approximated by the processes $X_{t}^{\varepsilon}$ that satisfy

$$
d X_{t}^{\varepsilon}=\left(\kappa(t) X_{t}^{\varepsilon}-\frac{1}{2 \lambda} X_{t}^{\varepsilon}-\frac{1}{2 \lambda \varepsilon} \beta\left(X_{t}^{\varepsilon}\right)\right) d t+\sqrt{\frac{1}{\lambda}} d B_{t} .
$$

Such a penalized version of reflected SDEs was studied in [9] in order to prove the existence of reflected diffusions in arbitrary convex bounded domains. We rather use 
(1.6) to define the extra-stress tensor corresponding to (1.4) as the limit of the stresses calculated for $X_{t}^{\varepsilon}$ via (1.2) with the penalized force (1.5) as $\varepsilon$ goes down to 0:

$$
\tau(t)=\frac{\eta_{p}}{\lambda}\left(\lim _{\varepsilon \rightarrow 0} \mathbb{E}\left(X_{t}^{\varepsilon} \otimes X_{t}^{\varepsilon}+\frac{1}{\varepsilon} X_{t}^{\varepsilon} \otimes \beta\left(X_{t}^{\varepsilon}\right)\right)-I\right) .
$$

To our best knowledge such a force law was not explored yet in the context of the modeling of polymeric fluids.

Let us mention another popular choice of $F($.$) , namely the FENE (Finitely Ex-$ tensible Non-linear Elastic) force [14]

$$
F_{\mathrm{FENE}}(X)=\frac{X}{1-|X|^{2} / R^{2}} .
$$

The existence of the solution $X_{t}$ to (1.1) with FENE force is proven in [7] and it is also shown that the process $X_{t}$ never leaves the ball $B(0, R)$ with probability 1 in the case $R \geq \sqrt{2}$. FENE force is a convenient approximation for a more complex inverse Langevin force law [13]. The latter is justified by considering the equilibrium distribution function for a Freely Jointed Chain. We argue however that the force derived from the potential (1.3) deserves also attention at least as a toy model since it can take into account the forces inside the rigid rods of a Freely Jointed Chain. Such a modeling can be found in $[6,5]$ where the rigid rods are replaced by very stiff (quasi-rigid) springs keeping the length almost constant. The force corresponding to (1.3) can be also an interesting alternative to the FENE one in applications of the dumbbell models in situations different from the dilute polymeric solutions. We cite for example the blood rheology model [11] where the dumbbells are used to represent the red blood cells and so a FENE force is not necessarily appropriate since the modeling is no longer based on a coarse graining procedure.

The main result of this article is the proof of the existence of the limit in (1.7) in the special case $\kappa=$ const. We give also a formula for this limit in terms of the solution to (1.4). The precise mathematical definitions and the statement of our Theorem are given in the next section. Section 3 contains the proof of our result. Finally, some preliminary numerical simulations illustrating our approach are given in section 4 .

\section{Formulation of the problem and main result}

We start with making precise definition of the problem. Let $R>0$ be the radius of the sphere $\overline{B(0, R)}$ in $\mathbb{R}^{d}$, where the process $X_{t}$ will remain and let $T>0$ be a final time. In order to simplify the notations we denote $\kappa-\frac{1}{2 \lambda} I$ by $\Gamma$ and $1 / \lambda$ by $\sigma^{2}$. The Skorohod problem is stated as follows (see [9]): given an initial condition $x \in B(0, R)$ and a $d \times d$ real matrix $\Gamma$, find continuous and progressively measurable $\mathbb{R}^{d}$-valued processes $X_{t}$ and $\mu_{t}$ such that

- $d X_{t}=\Gamma X_{t} d t-d \mu_{t}+\sigma d B_{t}, \quad X_{0}=x$,

- $X_{t} \in \overline{B(0, R)}$,

- $\mu_{t}$ has bounded variation on $[0, T]$ and $\mu_{0}=0$,

- for all $Z_{t}$ continuous an progressively measurable process taking

values in $\overline{B(0, R)}$ we have

$\int_{0}^{T}\left(X_{t}-Z_{t}\right) d \mu_{t} \geq 0, \forall T>0$.

Using the new notations, the penalized problem (1.6) reads (replacing $2 \lambda \varepsilon$ by $\varepsilon$ ): 
Given $\varepsilon>0$ and an initial condition $x \in B(0, R)$, find $X_{t}^{\varepsilon}$ satisfying

$$
d X_{t}^{\varepsilon}=\left(\Gamma X_{t}^{\varepsilon}-\frac{1}{\varepsilon} \beta\left(X_{t}^{\varepsilon}\right)\right) d t+\sigma d B_{t}, \quad X_{0}^{\varepsilon}=x .
$$

As already mentioned, system (2.1) and equation (2.2) has been studied in [9], where the two following results used in this paper are proven. For each $1 \leq p<\infty$, there exists a constant $C$ independent of $\varepsilon$ such that

$$
\mathbb{E}\left(\sup _{t \in[0, T]}\left|X_{t}^{\varepsilon}\right|^{p}\right) \leq C
$$

and for each $1 \leq p<\infty$ and $0<T<\infty$ we have

$$
\lim _{\varepsilon \rightarrow 0} \mathbb{E}\left(\sup _{0 \leq t \leq T}\left|X_{t}-X_{t}^{\varepsilon}\right|^{p}\right)=0 .
$$

We can now state the main theorem.

TheOREM 2.1. Let $R>0$ be the radius of the spherical domain and $T>0$ be a final time. Let $x \in B(0, R)$ be an initial condition and $\Gamma \in \mathbb{R}^{d \times d}$. Let $\left(X_{t}, \mu_{t}\right)$ be the solution of the reflecting $S D E$ (2.1) and $X_{t}^{\varepsilon}$ be the solution of (2.2). Then $\lim _{\varepsilon \rightarrow 0} \mathbb{E}\left(X_{t}^{\varepsilon} \otimes\right.$ $\left.\frac{1}{\varepsilon} \beta\left(X_{t}^{\varepsilon}\right)\right)$ exists for all $t \in[0, T]$ and is continuous in $t$. Moreover, $\frac{\partial}{\partial t} \mathbb{E}\left(X_{t} \otimes X_{t}\right)$ exists a.e. in $[0, T]$ and we have

$$
\begin{aligned}
\lim _{\varepsilon \rightarrow 0} \frac{1}{\varepsilon} \mathbb{E}\left(X_{t}^{\varepsilon} \otimes \beta\left(X_{t}^{\varepsilon}\right)\right)=-\frac{1}{2} \frac{\partial \mathbb{E}\left(X_{t} \otimes X_{t}\right)}{\partial t} & \\
& +\frac{1}{2} \mathbb{E}\left(\left(X_{t} \otimes X_{t}\right) \Gamma^{T}+\Gamma\left(X_{t} \otimes X_{t}\right)\right)+\frac{\sigma^{2}}{2} I,
\end{aligned}
$$

a.e. in $[0, T]$.

The proof of this theorem is postponed to the next section. We note here only that a weak form of this result, i.e. the corresponding formula for $\lim _{\varepsilon \rightarrow 0} \int_{0}^{t} \frac{1}{\varepsilon} \mathbb{E}\left(X_{s}^{\varepsilon} \otimes\right.$ $\left.\beta\left(X_{s}^{\varepsilon}\right)\right) d s$, is a simple consequence of Itô's rule applied to $X_{t}^{\varepsilon} \otimes X_{t}^{\varepsilon}$ and (2.4). The core of the proof will be thus to make sure that it is possible to change the order of limit and integration in this formula.

Corollary 2.2. Let $R>0$ be the radius of the spherical domain and $T>0$ be a final time. Let $x \in B(0, R)$ be an initial condition, $\kappa \in \mathbb{R}^{d \times d}, \lambda>0$ and $\eta_{p}>0$. Let $\left(X_{t}, \mu_{t}\right)$ be the solution of (1.4) in the sense of (2.1), $X_{t}^{\varepsilon}$ be the solution of (1.6), and

$$
\tau_{\varepsilon}=\frac{\eta_{p}}{\lambda}\left(\mathbb{E}\left(X_{t}^{\varepsilon} \otimes X_{t}^{\varepsilon}+X_{t}^{\varepsilon} \otimes \frac{1}{\varepsilon} \beta\left(X_{t}^{\varepsilon}\right)\right)-I\right) .
$$

Then the following limit is well-defined:

$$
\tau:=\lim _{\varepsilon \rightarrow 0} \tau_{\varepsilon} \in \mathcal{C}^{0}\left([0, T] ; \mathbb{R}^{d \times d}\right) .
$$

Moreover,

$$
\tau=-\eta_{p} \frac{\partial \mathbb{E}\left(X_{t} \otimes X_{t}\right)}{\partial t}+\eta_{p} \mathbb{E}\left(\left(X_{t} \otimes X_{t}\right) \kappa^{T}+\kappa\left(X_{t} \otimes X_{t}\right)\right)
$$


a.e. in $[0, T]$.

Proof. Set $\Gamma=\kappa-\frac{1}{2 \lambda} I, \sigma=\frac{1}{\sqrt{\lambda}}$ and apply Theorem 2.1 with $\varepsilon$ replaced back by $2 \lambda \varepsilon$.

We note that (2.7) is the well known Giesekus expression for the stress tensor in terms of the conformation tensor $\mathbb{E}\left(X_{t} \otimes X_{t}\right)$; see [2, p. 69, vol. II], for example. On the other hand, it is not clear how to directly write down the Kramers expression (1.2) of the stress in the model (1.6). Indeed, comparing (1.6) with the governing equation (1.1) of a general dumbbell model, we should interpret the spring force formally as

$$
F\left(X_{t}\right)=X_{t}+2 \lambda \frac{d \mu_{t}}{d t}
$$

so that a plausible expression for the stress inspired by (1.2) would be

$$
\tau=\frac{\eta_{p}}{\lambda}\left(\mathbb{E}\left(X_{t} \otimes X_{t}\right)+2 \lambda \lim _{\Delta t \rightarrow 0} \frac{1}{\Delta t} \mathbb{E}\left(X_{t} \otimes\left(\mu_{t+\Delta t}-\mu_{t}\right)\right)-I\right) .
$$

Whether this expression makes sense and whether it coincides with (2.7) is an open question.

\section{Proof of Theorem 2.1}

The proof of Theorem 2.1 will be presented as a sequence of lemmas, in which the crucial ingredient is the equicontinuity in $t$ of $\frac{1}{\varepsilon} \mathbb{E}\left(X_{t}^{\varepsilon} \otimes \beta\left(X_{t}^{\varepsilon}\right)\right)$ with respect to $\varepsilon$. More precisely, it will be shown in Lemma 3.4 that

$$
\lim _{h \rightarrow 0^{+}} \sup _{\varepsilon>0} \sup _{0 \leq t \leq T-h} \mathbb{E}\left(X_{t+h}^{\varepsilon} \otimes \frac{1}{\varepsilon} \beta\left(X_{t+h}^{\varepsilon}\right)-X_{t}^{\varepsilon} \otimes \frac{1}{\varepsilon} \beta\left(X_{t}^{\varepsilon}\right)\right)=0 .
$$

REMARK 3.1. Note that without Brownian motion, (3.1) does not hold in general. Indeed, let us consider the 1-dimensional variant $(d=1)$ of $(2.2)$ with $R=1, \Gamma=\gamma \in \mathbb{R}$, $\gamma>0, \sigma=0$, and $x \in] 0,1[$. Its solution can be written for $\varepsilon<1 / \gamma$ as

$$
X_{t}^{\varepsilon}= \begin{cases}x e^{\gamma t}, & t<\tilde{t}, \\ \left(1-\frac{1}{1-\varepsilon \gamma}\right) e^{(\gamma-1 / \varepsilon)(t-\tilde{t})}+\frac{1}{1-\varepsilon \gamma}, & t \geq \tilde{t},\end{cases}
$$

where $\tilde{t}=\frac{1}{\gamma} \ln \left(\frac{1}{x}\right)$. Let $h>0$, then it follows that

$$
\frac{1}{\varepsilon} X_{\tilde{t}+h}^{\varepsilon} \beta\left(X_{\tilde{t}+h}^{\varepsilon}\right)-\frac{1}{\varepsilon} X_{\tilde{t}}^{\varepsilon} \beta\left(X_{\tilde{t}}^{\varepsilon}\right)=\frac{1}{\varepsilon}\left(X_{\tilde{t}+h}^{\varepsilon}-X_{\tilde{t}}^{\varepsilon}\right)\left(X_{\tilde{t}+h}^{\varepsilon}+X_{\tilde{t}}^{\varepsilon}-1\right)
$$

and since $\lim _{\varepsilon \rightarrow 0} \frac{1}{\varepsilon}\left(X_{\tilde{t}+h}^{\varepsilon}-X_{\tilde{t}}^{\varepsilon}\right)=\gamma, \lim _{\varepsilon \rightarrow 0}\left(X_{\tilde{t}+h}^{\varepsilon}+X_{\tilde{t}}^{\varepsilon}\right)=2$ we obtain

$$
\lim _{h \rightarrow 0^{+}} \sup _{\varepsilon>0} \sup _{0 \leq t \leq T-h}\left|\frac{1}{\varepsilon} X_{t+h}^{\varepsilon} \beta\left(X_{t+h}^{\varepsilon}\right)-\frac{1}{\varepsilon} X_{t}^{\varepsilon} \beta\left(X_{t}^{\varepsilon}\right)\right| \geq \gamma>0 .
$$

Without loss of generality we now assume that $R=1$ and $\sigma=1$.

We shall need in what follows a special case of $(2.2)$ where $\Gamma=\gamma I, \gamma \in \mathbb{R}$. Let $y \in B(0,1)$ and consider the equation

$$
d Y_{t}^{\varepsilon}=\left(\gamma Y_{t}^{\varepsilon}-\frac{1}{\varepsilon} \beta\left(Y_{t}^{\varepsilon}\right)\right) d t+d B_{t}, \quad Y_{0}^{\varepsilon}=y,
$$


where

$$
\gamma=\sup _{|X|=1} X \cdot \Gamma X
$$

We establish first a comparison principle for the solutions of (2.2) and (3.2).

LEMma 3.1. Let $X_{t}^{\varepsilon}$ and $Y_{t}^{\varepsilon}$ be solutions of (2.2) and (3.2) with $|x| \leq|y|$. Then, for any nondecreasing function $g:[0, \infty[\rightarrow \mathbb{R}$

$$
\mathbb{E}^{x}\left(g\left(\left|X_{t}^{\varepsilon}\right|\right)\right) \leq \mathbb{E}^{y}\left(g\left(\left|Y_{t}^{\varepsilon}\right|\right)\right), \forall 0 \leq t \leq T
$$

Proof. Let $S(X)$ for any $X \in \mathbb{R}^{d}, X \neq 0, X \neq e_{1}$, be the orthogonal matrix that maps $X$ to $|X| e_{1}$ and leaves all the vectors orthogonal to $\left(X, e_{1}\right)$-plane unchanged. We set also $S(0)=S\left(e_{1}\right)=I$. It is easy to see that $\widetilde{B}_{t}=\int_{0}^{t} S\left(X_{s}\right) d B_{s}$ and $\widetilde{\widetilde{B}}_{t}=\int_{0}^{t} S\left(Y_{s}\right) d B_{s}$ are Brownian motions. Substitution to (2.2) and (3.2) gives

$$
\begin{aligned}
& d X_{t}^{\varepsilon}=\left(\Gamma X_{t}^{\varepsilon}-\frac{1}{\varepsilon} \beta\left(X_{t}^{\varepsilon}\right)\right) d t+S^{T}\left(X_{t}\right) d \widetilde{B}_{t} \\
& d Y_{t}^{\varepsilon}=\left(\gamma Y_{t}^{\varepsilon}-\frac{1}{\varepsilon} \beta\left(Y_{t}^{\varepsilon}\right)\right) d t+S^{T}\left(Y_{t}\right) d \widetilde{\widetilde{B}}_{t}
\end{aligned}
$$

Since the statement of the lemma involves only the expectations of $g\left(\left|X_{t}^{\varepsilon}\right|\right)$ and $g\left(\left|Y_{t}^{\varepsilon}\right|\right)$ we are free to change the realizations of the Brownian motions $\tilde{B}_{t}$ and $\tilde{\tilde{B}}_{t}$. We choose them to be the same Brownian motion, which will be denoted again as $B_{t}=$ $\left(B_{t}^{1}, \ldots, B_{t}^{d}\right)$. Itô's rule gives then

$$
\begin{gathered}
d\left|X_{t}^{\varepsilon}\right|^{2}=2\left(X_{t}^{\varepsilon} \cdot \Gamma X_{t}^{\varepsilon}-X_{t}^{\varepsilon} \cdot \frac{1}{\varepsilon} \beta\left(X_{t}^{\varepsilon}\right)+\frac{d}{2}\right) d t+2\left|X_{t}^{\varepsilon}\right| d B_{t}^{1} \\
d\left|Y_{t}^{\varepsilon}\right|^{2}=2\left(\gamma\left|Y_{t}^{\varepsilon}\right|^{2}-Y_{t}^{\varepsilon} \cdot \frac{1}{\varepsilon} \beta\left(Y_{t}^{\varepsilon}\right)+\frac{d}{2}\right) d t+2\left|Y_{t}^{\varepsilon}\right| d B_{t}^{1}
\end{gathered}
$$

We denote $\Delta_{t}=\left|X_{t}^{\varepsilon}\right|^{2}-\left|Y_{t}^{\varepsilon}\right|^{2}$ and reproduce the proof of [8, Chapter 5, Proposition 2.18]. Choose a nondecreasing sequence of functions $\phi_{n} \in C^{2}(\mathbb{R})$ such that $\operatorname{supp} \phi_{n} \in$ $(0, \infty)$ and for all $z>0$ we have $\lim _{n \rightarrow \infty} \phi_{n}(z)=z, 0 \leq \phi_{n}^{\prime}(z) \leq 1$, and $\left|\phi_{n}^{\prime \prime}(z)\right| \leq 2 /(n z)$. We have from (3.6), (3.7) by Itô's rule that

$$
\begin{array}{r}
\mathbb{E}\left(\phi_{n}\left(\Delta_{t}\right)\right)=\mathbb{E} \int_{0}^{t}\left(2 \phi_{n}^{\prime}\left(\Delta_{s}\right)\left(X_{s}^{\varepsilon} \cdot \Gamma(s) X_{s}^{\varepsilon}-\gamma\left|Y_{s}^{\varepsilon}\right|^{2}-f_{\varepsilon}\left(\left|X_{s}^{\varepsilon}\right|^{2}\right)+f_{\varepsilon}\left(\left|Y_{s}^{\varepsilon}\right|^{2}\right)\right) d s\right. \\
\left.+\mathbb{E} \int_{0}^{t} \phi_{n}^{\prime \prime}\left(\Delta_{s}\right)\left(\left|X_{s}^{\varepsilon}\right|-\left|Y_{s}^{\varepsilon}\right|\right)^{2}\right) d s
\end{array}
$$

where $f_{\varepsilon}(z)=1_{(1, \infty)}(z)(z-\sqrt{z}) / \varepsilon$ is Lipschitz with constant $1 / \varepsilon$. Noting that $\left.\left|\phi_{n}^{\prime \prime}\left(\Delta_{t}\right)\right|\left(\left|X_{t}^{\varepsilon}\right|-\left|Y_{t}^{\varepsilon}\right|\right)^{2}\right) \leq\left|\phi_{n}^{\prime \prime}\left(\Delta_{t}\right)\right|\left|\Delta_{t}\right| \leq 2 / n$ we obtain

$$
\mathbb{E}\left(\phi_{n}\left(\Delta_{t}\right)\right) \leq \mathbb{E} \int_{0}^{t}\left(\gamma+\frac{1}{\varepsilon}\right) \Delta_{s}^{+} d s+\frac{2 t}{n}
$$


where $\Delta_{t}^{+}=\Delta_{t} \vee 0$. Taking the limit and applying Gronwall lemma gives $\Delta_{t}^{+}=0$, hence $\left|X_{t}^{\varepsilon}\right| \leq\left|Y_{t}^{\varepsilon}\right|$ a.s. and (3.3) holds.

The following uniform bound in $\varepsilon$ on the approximated stress tensor is crucial to prove Theorem 2.1.

Lemma 3.2. For all $r \in[0,1)$ there exists finite $C(r)$ so that for $X_{t}^{\varepsilon}$ the solution of (2.2) for any $x \in B(0, r)$ and $t \in[0, T]$, we have

$$
\mathbb{E}^{x}\left(X_{t}^{\varepsilon} \cdot \frac{1}{\varepsilon} \beta\left(X_{t}^{\varepsilon}\right)\right) \leq C(r),
$$

where $C(r)$ is a constant only depending on $r$ and $\gamma$.

Proof. Denote $\pi_{\varepsilon}$ to be the stationary distribution of (3.2) and $\pi_{\varepsilon} \cap I_{+}$to be the distribution $\pi_{\varepsilon}$ conditioned on the set $I_{+}=\left\{z \in \mathbb{R}^{d}:|z|>r\right\}$. Lemma 3.1 with $g(X)=X \cdot \frac{1}{\varepsilon} \beta(X)$ entails

$$
\mathbb{E}^{x}\left(X_{t}^{\varepsilon} \cdot \frac{1}{\varepsilon} \beta\left(X_{t}^{\varepsilon}\right)\right) \leq \mathbb{E}^{\pi_{\varepsilon} \cap I_{+}}\left(Y_{t}^{\varepsilon} \cdot \frac{1}{\varepsilon} \beta\left(Y_{t}^{\varepsilon}\right)\right) \leq \frac{\mathbb{E}^{\pi_{\varepsilon}}\left(Y_{t}^{\varepsilon} \cdot \frac{1}{\varepsilon} \beta\left(Y_{t}^{\varepsilon}\right)\right)}{\pi_{\varepsilon}\left(I_{+}\right)} .
$$

Itô's rule for $\left|Y_{t}^{\varepsilon}\right|^{2}$ gives

$$
\mathbb{E}^{\pi_{\varepsilon}}\left(Y_{t}^{\varepsilon} \cdot \frac{1}{\varepsilon} \beta\left(Y_{t}^{\varepsilon}\right)\right)=\gamma \mathbb{E}^{\pi_{\varepsilon}}\left(\left|Y_{t}^{\varepsilon}\right|^{2}\right)+\frac{d}{2} .
$$

The stationary distribution is easily found as $\pi_{\varepsilon}(Y)=\frac{1}{C_{\varepsilon}} \phi_{\varepsilon}(Y)$ with

$$
\phi_{\varepsilon}(Y)=\left\{\begin{array}{l}
e^{\gamma|Y|^{2}},|Y|<1 \\
e^{\gamma|Y|^{2}-\frac{(|Y|-1)^{2}}{\varepsilon},}, \text { otherwise }
\end{array}\right.
$$

and $C_{\varepsilon}=\int_{\mathbb{R}^{d}} \phi_{\varepsilon}(Y) d Y$. We see now by the dominated convergence theorem that the limits $\lim _{\varepsilon \rightarrow 0} \mathbb{E}^{\pi_{\varepsilon}}\left(\left|Y_{t}^{\varepsilon}\right|^{2}\right)$ and $\lim _{\varepsilon \rightarrow 0} \pi_{\varepsilon}\left(I^{+}\right)$exist and are finite. Hence $\mathbb{E}^{x}\left(X_{t}^{\varepsilon}\right.$. $\left.\frac{1}{\varepsilon} \beta\left(X_{t}^{\varepsilon}\right)\right)$ is bounded uniformly in $\varepsilon$ by (3.8) and (3.9).

Corollary 3.3. Take any $x \in B(0,1)$ and any time $t \in[0, T]$. The following estimates for the solution $X_{t}^{\varepsilon}$ of (2.2) hold for all $1 \leq i, j \leq d$

$$
\mathbb{E}^{x}\left(\left|\left(X_{t}^{\varepsilon}\right)_{i} \frac{1}{\varepsilon} \beta\left(X_{t}^{\varepsilon}\right)_{j}\right|\right) \leq C(|x|)
$$

with $C(|x|)$ independent of $\varepsilon$.

Proof. Immediate since $\left|X_{i}(\beta(X))_{j}\right| \leq X \cdot \beta(X)$ for any $X \in \mathbb{R}^{d}$.

We now prove (3.1) in a more general case.

Lemma 3.4. Let $X_{t}^{\varepsilon}$ be the solution of (2.2) with $x \in B(0,1)$ and $\phi: \mathbb{R}^{d} \rightarrow \mathbb{R}^{+}$such that $\phi(z)=0$ for all $z \in B(0,1)$. Suppose that for all $t \in[0, T]$ it holds that

$$
\mathbb{E}^{x}\left|\frac{1}{\varepsilon} \phi\left(X_{t}^{\varepsilon}\right)\right| \leq C(|x|)
$$

with $C(|x|)$ independent of $\varepsilon$. Then we have

$$
\lim _{h \rightarrow 0^{+}} \sup _{\varepsilon>0} \sup _{0 \leq t \leq T-h}\left|\mathbb{E}^{x}\left(\frac{1}{\varepsilon}\left(\phi\left(X_{t+h}^{\varepsilon}\right)-\phi\left(X_{t}^{\varepsilon}\right)\right)\right)\right|=0 .
$$


Proof. Fix any number $r$ so that $|x|<r<1$. Using the Markov property we have for any $h>0, t \geq h^{\alpha}$ with $\alpha \in(0,1)$ to be chosen later

$$
\begin{aligned}
\mathbb{E}^{x}\left(\frac{1}{\varepsilon} \phi\left(X_{t}^{\varepsilon}\right)\right)= & \int_{B(0, r)} p_{h^{\alpha}}^{X^{\varepsilon}}(z) \mathbb{E}^{z}\left(\frac{1}{\varepsilon} \phi\left(X_{t-h^{\alpha}}^{\varepsilon}\right)\right) d z \\
& +\int_{\mathbb{R}^{d} \backslash B(0, r)} p_{h^{\alpha}}^{X^{\varepsilon}}(z) \mathbb{E}^{z}\left(\frac{1}{\varepsilon} \phi\left(X_{t-h^{\alpha}}^{\varepsilon}\right)\right) d z
\end{aligned}
$$

where $p_{a}^{Z}$ denotes the probability density of a process $Z$ at time $a$. We introduce the stopping time $\sigma=\inf \left\{s>0:\left|X_{s}^{\varepsilon}\right|=r\right\}$. The second integral in the above equality can be now rewritten using the strong Markov property and hypothesis (3.10) as

$$
\begin{aligned}
\mathbb{E}^{x}\left(\frac{1}{\varepsilon} \phi\left(X_{t}^{\varepsilon}\right) 1_{\left|X_{h^{\alpha}}\right| \geq r}\right) & \leq \mathbb{E}^{x}\left(\frac{1}{\varepsilon} \phi\left(X_{t}^{\varepsilon}\right) 1_{\sigma \leq h^{\alpha}}\right)=\mathbb{E}^{x}\left(\mathbb{E}^{x}\left(\frac{1}{\varepsilon} \phi\left(X_{t}^{\varepsilon}\right) 1_{\sigma \leq h^{\alpha}} \mid \mathcal{F}_{\sigma}\right)\right) \\
& =\mathbb{E}^{x}\left(\mathbb{E}^{X_{\sigma}^{\varepsilon}}\left(\frac{1}{\varepsilon} \phi\left(X_{t-\sigma}^{\varepsilon}\right)\right) 1_{\sigma \leq h^{\alpha}}\right) \leq C(r) \mathcal{P}\left(\sigma \leq h^{\alpha}\right) .
\end{aligned}
$$

Consider now the unconstrained processes $X$

$$
d X_{t}=\Gamma X_{t} d t+d B_{t}, \quad X_{0}=x .
$$

We see that $X_{t}^{\varepsilon}$ and $X_{t}$ coincide for time $t$ up to $\sigma$, hence this stopping time can be redefined as $\sigma=\inf \left\{s>0:\left|X_{s}\right|=r\right\}$ and the probability $\mathcal{P}\left(\sigma \leq h^{\alpha}\right)$ is obviously independent of $\varepsilon$ and moreover vanishes in the limit $h \rightarrow 0$. Indeed, this is easy to see in the special case $\Gamma=0$ when $X_{t}$ is just a Brownian motion started at $x$, see for instance [8, Equ. (6.3) p. 80]. To prove that $\mathcal{P}\left(\sigma \leq h^{\alpha}\right) \rightarrow 0$ as $h \rightarrow 0$ for $X_{t}$ with arbitrary $\Gamma$ one can invoke the Girsanov theorem to pass from $B_{t}$ to $X_{t}$. We will denote the quantities that can be bounded by a function of $h$ independent of $\varepsilon$ and vanishing as $h \rightarrow 0$ by $o(1)$. Hence, owning (3.13), we can rewrite (3.12) as

$$
\mathbb{E}^{x}\left(\frac{1}{\varepsilon} \phi\left(X_{t}^{\varepsilon}\right)\right)=\int_{B(0, r)} p_{h^{\alpha}}^{X^{\varepsilon}}(z) \mathbb{E}^{z}\left(\frac{1}{\varepsilon} \phi\left(X_{t-h^{\alpha}}^{\varepsilon}\right)\right) d z+o(1) .
$$

We now apply similar ideas to estimate the first integral in (3.12) via the same integral in which the probability density of $X^{\varepsilon}$ is replaced by that of $X$. We do it first on only a part of $B(0, r): B_{r}^{+}=\left\{z \in B(0, r): p_{h^{\alpha}}^{X^{\varepsilon}}(z)>p_{h^{\alpha}}^{X}(z)\right\}$.

$$
\begin{aligned}
& \left|\int_{B_{r}^{+}} p_{h^{\alpha}}^{X^{\varepsilon}}(z) \mathbb{E}^{z}\left(\frac{1}{\varepsilon} \phi\left(X_{t-h^{\alpha}}^{\varepsilon}\right)\right) d z-\int_{B_{r}^{+}} p_{h^{\alpha}}^{X}(z) \mathbb{E}^{z}\left(\frac{1}{\varepsilon} \phi\left(X_{t-h^{\alpha}}^{\varepsilon}\right)\right) d z\right| \\
& \leq \int_{B_{r}^{+}}\left(p_{h^{\alpha}}^{X^{\varepsilon}}(z)-p_{h^{\alpha}}^{X}(z)\right) \mathbb{E}^{z}\left|\frac{1}{\varepsilon} \phi\left(X_{t-h^{\alpha}}^{\varepsilon}\right)\right| d z \leq C(r) \int_{B_{r}^{+}}\left(p_{h^{\alpha}}^{X^{\varepsilon}}(z)-p_{h^{\alpha}}^{X}(z)\right) d z \\
& =C(r)\left[\mathcal{P}\left(X_{h^{\alpha}}^{\varepsilon} \in B_{r}^{+}\right)-\mathcal{P}\left(X_{h^{\alpha}} \in B_{r}^{+}\right)\right] \\
& =C(r)\left[\mathcal{P}\left(\left\{X_{h^{\alpha}}^{\varepsilon} \in B_{r}^{+}\right\} \cap\left\{\sigma \leq h^{\alpha}\right\}\right)-\mathcal{P}\left(\left\{X_{h^{\alpha}} \in B_{r}^{+}\right)\right\} \cap\left\{\sigma \leq h^{\alpha}\right\}\right. \\
& \left.\quad+\mathcal{P}\left(\left\{X_{h^{\alpha}}^{\varepsilon} \in B_{r}^{+}\right\} \cap\left\{\sigma \geq h^{\alpha}\right\}\right)-\mathcal{P}\left(\left\{X_{h^{\alpha}} \in B_{r}^{+}\right)\right\} \cap\left\{\sigma \geq h^{\alpha}\right\}\right] \\
& \quad\left(\text { using the fact that } X_{t}^{\varepsilon} \text { and } X_{t} \text { are the same for } t \leq \sigma\right) \\
& =C(r)\left[\mathcal{P}\left(\left\{X_{h^{\alpha}}^{\varepsilon} \in B_{r}^{+}\right\} \cap\left\{\sigma \leq h^{\alpha}\right\}\right)-\mathcal{P}\left(\left\{X_{h^{\alpha}} \in B_{r}^{+}\right)\right\} \cap\left\{\sigma \leq h^{\alpha}\right\}\right] \\
& \leq C(r) \mathcal{P}\left(\sigma \leq h^{\alpha}\right)=o(1) .
\end{aligned}
$$


Applying the same arguments to the integrals over $B_{r}^{-}=B(0, r) \backslash B_{r}^{+}$yields

$$
\int_{B(0, r)} p_{h^{\alpha}}^{X^{\varepsilon}}(z) \mathbb{E}^{z}\left(\frac{1}{\varepsilon} \phi\left(X_{t-h^{\alpha}}^{\varepsilon}\right)\right) d z=\int_{B(0, r)} p_{h^{\alpha}}^{X}(z) \mathbb{E}^{z}\left(\frac{1}{\varepsilon} \phi\left(X_{t-h^{\alpha}}^{\varepsilon}\right)\right) d z+o(1) .
$$

Hence

$$
\mathbb{E}^{x}\left(\frac{1}{\varepsilon} \phi\left(X_{t}^{\varepsilon}\right)\right)=\int_{B(0, r)} p_{h^{\alpha}}^{X}(z) \mathbb{E}^{z}\left(\frac{1}{\varepsilon} \phi\left(X_{t-h^{\alpha}}^{\varepsilon}\right)\right) d z+o(1) .
$$

We now rewrite the last relation with $t$ replaced by $t+h$ and $h^{\alpha}$ replaced by $h^{\alpha}+h$ and take the difference of the two. This yields

$$
\begin{aligned}
\mid \mathbb{E}^{x} & \left(\frac{1}{\varepsilon} \phi\left(X_{t+h}^{\varepsilon}\right)\right)-\mathbb{E}^{x}\left(\frac{1}{\varepsilon} \phi\left(X_{t}^{\varepsilon}\right)\right) \mid \\
& =\left|\int_{B(0, r)}\left(p_{h^{\alpha}+h}^{X}(z)-p_{h^{\alpha}}^{X}(z)\right) \mathbb{E}^{z}\left(\frac{1}{\varepsilon} \phi\left(X_{t-h^{\alpha}}^{\varepsilon}\right)\right) d z\right|+o(1) \\
& \leq C(r) \int_{B(0, r)}\left|p_{h^{\alpha}+h}^{X}(z)-p_{h^{\alpha}}^{X}(z)\right| d z+o(1) .
\end{aligned}
$$

A direct calculation of $p_{t}^{X}$ allows us to show that

$$
\left|p_{h^{\alpha}+h}^{X}(z)-p_{h^{\alpha}}^{X}(z)\right| \leq C h^{\delta} \text { for } z \in B(0,1), 0 \leq h \leq 1
$$

for any $0<\alpha<\frac{2}{d+2}, \delta=1-\frac{\alpha(d+2)}{2}>0$ and a constant $C$ that depends only on $\Gamma$ and $\alpha$. Indeed, $X_{t}$ is a Gaussian process with the mean $m_{t}=\exp (\Gamma t) x$ and the covariance matrix $\Sigma_{t}$ satisfying the differential equation

$$
\Sigma_{t}^{\prime}=\Gamma \Sigma_{t}+\Sigma_{t} \Gamma^{T}+I, \quad \Sigma_{0}=0
$$

Thus,

$$
p_{t}^{X}(z)=\frac{1}{(2 \pi)^{d / 2} \sqrt{\operatorname{det}\left(\Sigma_{t}\right)}} \exp \left(-\frac{1}{2}\left(z-m_{t}\right) \cdot \Sigma_{t}^{-1}\left(z-m_{t}\right)\right) .
$$

The differential equation above implies

$$
\Sigma_{t}=t I+o(t), t \rightarrow 0 \text { and } \Sigma_{h^{\alpha}+h}=\Sigma_{h^{\alpha}}\left(I+h^{1-\alpha} I+o\left(h^{1-\alpha}\right)\right), h \rightarrow 0
$$

so that

$$
\frac{1}{\sqrt{\operatorname{det} \Sigma_{h^{\alpha}}}}-\frac{1}{\sqrt{\operatorname{det} \Sigma_{h^{\alpha}+h}}}=\frac{1}{\sqrt{\operatorname{det}\left(\Sigma_{h^{\alpha}}\right)}}\left[1-\sqrt{\operatorname{det}\left(\Sigma_{h^{\alpha}+h}^{-1} \Sigma_{h^{\alpha}}\right)}\right]=O\left(h^{1-\alpha-\alpha \frac{d}{2}}\right) .
$$

Similar calculations show that

$$
m_{h^{\alpha}+h}=(\Gamma h+o(h)) m_{h^{\alpha}} \text { and } \Sigma_{h^{\alpha}+h}^{-1}=\left(I-h^{1-\alpha} I+o\left(h^{1-\alpha}\right)\right) \Sigma_{h^{\alpha}}^{-1},
$$

hence

$$
\begin{aligned}
\exp \left(-\frac{1}{2}\left(z-m_{h^{\alpha}+h}\right) \cdot \Sigma_{h^{\alpha}+h}^{-1}\left(z-m_{h^{\alpha}+h}\right)\right) & =\exp \left(-\frac{1}{2}\left(z-m_{h^{\alpha}}\right) \cdot \Sigma_{h^{\alpha}}^{-1}\left(z-m_{h^{\alpha}}\right)\right) \\
& +O\left(h^{1-\alpha}\right) .
\end{aligned}
$$


The symbol $O\left(h^{s}\right)$ stands here for a function of $h$ and $z$ bounded by $C h^{s}$ on $(h, z) \in$ $[0,1] \times \overline{B(0,1)}$. Combining these estimates gives

$$
p_{h^{\alpha}+h}^{X}(z)-p_{h^{\alpha}}^{X}(z)=O\left(h^{1-\alpha-\alpha \frac{d}{2}}\right)=O\left(h^{\delta}\right) .
$$

This is exactly (3.14), therefore the Lemma is proved.

We are now able to prove Theorem 2.1.

Proof. (of Theorem 2.1.) Let $\left(\varepsilon_{n}\right)_{n \geq 1} \subset \mathbb{R}$ be a sequence with $\lim _{n \rightarrow \infty} \varepsilon_{n}=0$ and $1 \leq i, j \leq d$. We denote $f_{n}(t)=\frac{1}{\varepsilon_{n}} \mathbb{E}\left(\left(X_{t}^{\varepsilon_{n}}\right)_{i}\left(\beta\left(X_{t}^{\varepsilon_{n}}\right)\right)_{j}\right)$ to be the $i j$-th component of the tensor $\frac{1}{\varepsilon_{n}} \mathbb{E}\left(X_{t}^{\varepsilon_{n}} \otimes \beta\left(X_{t}^{\varepsilon_{n}}\right)\right)$. Itô's rule gives that for all $t \in[0, T]$ and for all $n \geq 1$

$$
\begin{aligned}
\int_{0}^{t} f_{n}(s) d s=-\frac{1}{2}\left(\mathbb{E}\left(\left(X_{t}^{\varepsilon_{n}}\right)_{i}\left(X_{t}^{\varepsilon_{n}}\right)_{j}\right)-x_{i} x_{j}\right) \\
\left.+\frac{1}{2}\left(\int_{0}^{t} \mathbb{E}\left(X_{s}^{\varepsilon_{n}} \otimes X_{s}^{\varepsilon_{n}}\right) \Gamma^{T}+\Gamma\left(X_{s}^{\varepsilon_{n}} \otimes X_{s}^{\varepsilon_{n}}\right)\right) d s\right)_{i j}+\frac{1}{2} t \delta_{i j} .
\end{aligned}
$$

Let

$$
\begin{aligned}
g(t)=-\frac{1}{2}\left(\mathbb{E}\left(\left(X_{t}\right)_{i}\left(X_{t}\right)_{j}\right)-\right. & \left.x_{i} x_{j}\right) \\
& \left.+\frac{1}{2}\left(\int_{0}^{t} \mathbb{E}\left(X_{s} \otimes X_{s}\right) \Gamma^{T}+\Gamma\left(X_{s} \otimes X_{s}\right)\right) d s\right)_{i j}+\frac{1}{2} t \delta_{i j} .
\end{aligned}
$$

Using (2.4) we obtain

$$
\lim _{n \rightarrow \infty} \int_{0}^{t} f_{n}(s) d s=g(t) .
$$

On the other hand, Corollary 3.3 implies that the sequence $\left(f_{n}(t)\right)_{n \geq 1}$ is equibounded. Then Lemma 3.4 applies with $\phi(X)=X_{i} \beta(X)_{j}$ (cf. Corollary 3.3) and ensures that the sequence $\left(f_{n}(t)\right)_{n \geq 1}$ is equicontinuous. By Ascoli-Arzelà's theorem, there exists a subsequence $\left(f_{n_{k}}\right)_{k \geq 1}$ converging in $\mathcal{C}^{0}([0, T] ; \mathbb{R})$. Let us denote this limit by $f \in$ $\mathcal{C}^{0}([0, T] ; \mathbb{R})$ and note that the uniform convergence implies

$$
\lim _{k \rightarrow \infty} \int_{0}^{t} f_{n_{k}}(s) d s=\int_{0}^{t} f(s) d s=g(t)
$$

Hence, $g^{\prime}(t)$ exists in $[0, T]$ and $f(t)=g^{\prime}(t)$ for all $t \in[0, T]$. Going back to (3.15), we see that this reasoning is independent of the choice of the sequence $\left(\varepsilon_{n}\right)_{n \geq 1}$ and in particular the limit of any subsequence converging in $\mathcal{C}^{0}([0, T] ; \mathbb{R})$ is $f(t)=g^{\prime}(t)$. This means that $\lim _{\varepsilon \rightarrow 0} \mathbb{E}\left(\left(X_{t}^{\varepsilon}\right)_{i}\left(\beta\left(X_{t}^{\varepsilon}\right)\right)_{j}\right)$ exists in $\mathcal{C}^{0}([0, T] ; \mathbb{R})$ and is equal to $g^{\prime}(t)$, which proves (2.5) a.e in $[0, T]$.

\section{Numerical simulations}

In this section we present and computationally study three numerical schemes for the model (2.2) leading to a stochastic approximation of the extra-stress tensor $\tau$ given by (2.6) or (2.7). We note that no theoretical analysis has been done yet for the validity of these approximations. Hence we should compare them with some more reliable results, which can be provided by simulations based on the PDE for the probability density function of the process $X_{t}$, that is the Fokker-Planck equation. 
The stress tensor provided by the latter is then used as reference solution to compare the performances of the different stochastic schemes.

The results reported below correspond to the following physical setting: shear flow in $2 \mathrm{D}$ with

$$
\kappa=\left(\begin{array}{cc}
0 & \dot{\gamma} \\
0 & 0
\end{array}\right)
$$

and $\dot{\gamma}=10, \lambda=\eta_{p}=1, R=\sqrt{20}$. Moreover, the initial distribution of $X$ is taken in all our experiments to be the equilibrium one, that is the stationary distribution of the model $(2.2)$ with $\kappa=0$, which is the normal distribution $\mathcal{N}(0,1)$ restricted to the ball $\overline{B(0, R)}$.

4.1. The Fokker-Planck method. Let $\psi(t, X), X \in B(B=B(0, R))$ be the probability density of the process $X_{t}$. It is well known that $\psi$ satisfies the following Fokker-Planck equation

$$
\frac{\partial \psi}{\partial t}+\operatorname{div}_{X}\left(\left(\kappa X-\frac{1}{2 \lambda} X\right) \psi\right)=\frac{1}{2 \lambda} \Delta_{X} \psi
$$

with the boundary conditions of probability flux zero:

$$
\left.\left(-\kappa X \psi+\frac{1}{2 \lambda} X \psi+\frac{1}{2 \lambda} \nabla_{X} \psi\right) \cdot \mathbf{n}\right|_{\partial B}=0 .
$$

The expression for the extra-stress tensor can be rewritten in terms of the probability density and using (4.1)-(4.2) as

$$
\begin{aligned}
\tau & =-\eta_{p} \frac{\partial}{\partial t} \int_{B} X \otimes X \psi d X+\eta_{p} \int_{B}\left(\kappa X \otimes X+X \otimes X \kappa^{T}\right) \psi d X \\
& =\frac{\eta_{p}}{\lambda} \int_{B} X \otimes X \psi d X+\frac{\eta_{p}}{\lambda R} \int_{B} X \otimes X \psi d s-\frac{\eta_{p}}{\lambda} I
\end{aligned}
$$

In order to construct the numerical method we introduce the polar coordinates $r \in[0, R], \theta \in[0,2 \pi)$ and perform the change of variables $r \rightarrow \eta$ given by $r^{2}=\frac{1+\eta}{2}$, $\eta \in[0,1]$, which takes into account the fact that $\psi(t, X)$ is an even function of $X$ as long as the initial condition $\psi(t, X)$ is even, which is the case in our numerical experiments. We introduce the Gauss-Legendre-Radau collocation points on $[-1,1]$ (including 1 and excluding -1) and denote $h_{i}(\eta)$ to be the corresponding Lagrange inerpolating polynomials. We then approximate $\psi(t, X)=\psi(t, \eta, \theta)$ in $\eta, \theta$ by

$$
\psi(t, \eta, \theta) \approx \sum_{i=0}^{N_{R}} \sum_{j=0}^{N_{F}} \psi_{i j}^{0}(t) h_{i}(\eta) \cos 2 j \theta+\sum_{i=0}^{N_{R}} \sum_{j=1}^{N_{F}} \psi_{i j}^{1}(t) h_{i}(\eta) \sin 2 j \theta .
$$

Note that -1 is not included in the set of collocation points because the boundary condition at $\eta=-1$ (i.e. $r=0$ ) is already satisfied by the choice of $\eta$. We plug then this approximation into the variational formulation of the Fokker-Planck equation and obtain the linear system for the coefficients $\left\{\psi_{i j}^{0}(t), \psi_{i j}^{1}(t)\right\}$ by the Galerkin method. The system is then discretized in time by implicit Euler scheme. The numerical results are presented at figure 4.1. They demonstrate that the method converges under refinement in space and time. We take the numerical solution at the finest level as the reference one in the following simulations. 


\begin{tabular}{c|ccc} 
& $N_{R}$ & $N_{F}$ & $\Delta t$ \\
\hline Level 3 & 8 & 4 & $0.1 \times 2^{-3}$ \\
Level 4 & 12 & 8 & $0.1 \times 2^{-4}$ \\
Level 5 & 20 & 12 & $0.1 \times 2^{-5}$ \\
Level 6 & 26 & 16 & $0.1 \times 2^{-6}$
\end{tabular}

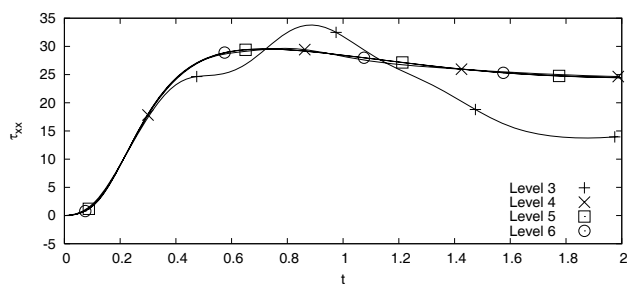

FIG. 4.1. The left part of the figure describes the numerical parameters corresponding to different discretization levels. The right part of the figure presents the evolution of $\tau_{x x}$ (Top: $t \in[0,2]$, Bottom: $t \in[1.4,2])$.

4.2. Stochastic simulations. We will consider three methods to discretize (1.4) in time. We introduce the uniform grid in time $t_{n}=n \Delta t$ and will denote the approximation for $X_{t_{n}}$ by $X_{n}^{\Delta t}$. The number of random realizations used to approximate the expectation is denoted by $M$. For all the simulations, we impose the relation $M \propto(\Delta t)^{2}$ to ensure that the stochastic noise does not affect the convergence order with respect to the time step.

4.2.1. The penalization method. The first method is the algorithm by penalization, which is a direct discretization of the penalized SDE (1.6)

$$
X_{n+1}^{\Delta t}-X_{n}^{\Delta t}=\left(\kappa\left(t_{n}\right) X_{n}^{\Delta t}-\frac{1}{2 \lambda} X_{n}^{\Delta t}-\frac{1}{2 \lambda \varepsilon} \beta\left(X_{n}^{\Delta t}\right)\right) \Delta t+\sqrt{\frac{1}{\lambda}}\left(B_{t_{n+1}}-B_{t_{n}}\right) .
$$

The tensor $\tau$ at time $t_{n}$ is then approximated by

$$
\tau_{n}^{\Delta t}=\frac{\eta_{p}}{\lambda} \mathbb{E}\left(X_{n}^{\Delta t} \otimes X_{n}^{\Delta t}+\frac{1}{\varepsilon} X_{n}^{\Delta t} \otimes \beta\left(X_{n}^{\Delta t}\right)\right)-\frac{\eta_{p}}{\lambda} I .
$$

The choice of penalization parameter $\varepsilon$ is a delicate question. We know from [12] that the scheme (4.3) has weak convergence of order $\sqrt{\Delta t}$ provided $\varepsilon \geq \Delta t$. This result is insufficient, however, to conclude the convergence of the approximation of the stress (4.4). Experimentally, we have tested the two choices for $\varepsilon$, namely $\varepsilon=$ $\sqrt{\Delta t}$ and $\varepsilon=\Delta t$. We found that the convergence with respect to the $L^{2}$-norm of $\tau$, $\sqrt{\int_{0}^{T}\left(\tau_{x x}^{2}+\tau_{x y}^{2}+\tau_{y y}^{2}\right)}$, is of order $\sqrt{\Delta t}$ and $\Delta t$ respectively (see figure 4.5). Therefore, we adopt the latter choice and report in figure 4.2 the evolution of the $x x$ component of the extra stress tensor $\tau$ along with its variance. The variance is approximated as follows. The stochastic simulation is performed 100 times, each time using $M$ samples generated with the independent sets of Brownian increments $\left(B_{t_{n+1}}-B_{t_{n}}\right)$. Each calculation yields an approximation of the extra stress $\tau_{n}^{\Delta t,(k)}$ where $k=1, \ldots, 100$ stands for the simulation number. Then, the variance is defined as

$$
\operatorname{Var}\left(\tau_{i j}\right)\left(t_{n}\right)=\frac{1}{100} \sum_{i=1}^{100}\left(\tau_{n, i j}^{\Delta t,(k)}-\overline{\tau_{n, i j}^{\Delta t}}\right)^{2} .
$$

where $\overline{\tau_{n, i j}^{\Delta t}}=\frac{1}{100} \sum_{k=1}^{100} \tau_{n, i j}^{\Delta t,(k)}$. The convergence of the penalization method to the Fokker-Planck solution is clearly observed in figure 4.2. 

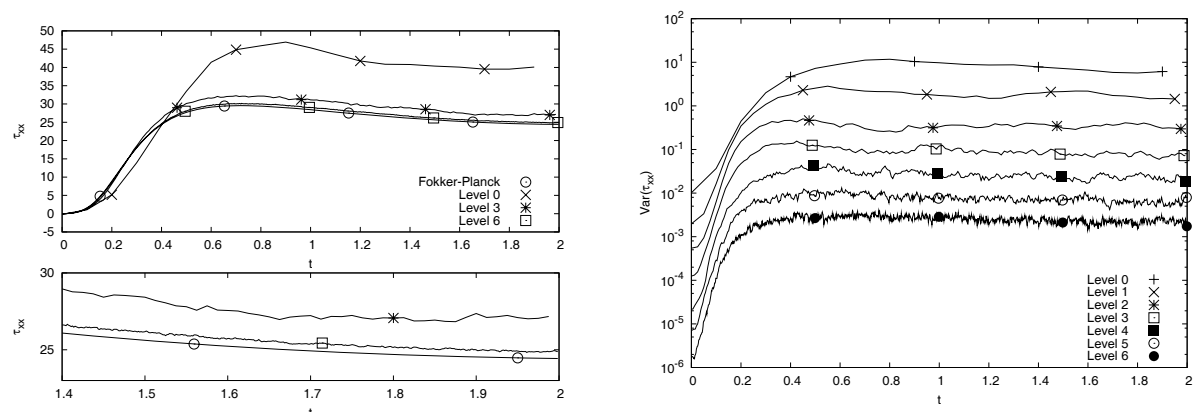

FIG. 4.2. The penalization method in shear flow with $\dot{\gamma}=10$. Seven different discretization levels $L \in\{0,1,2,3,4,5,6\}$ are considered. For each level $L$, the corresponding numerical parameters are $\Delta t_{L}=0.1 \times 2^{-L}$ and $M_{L}=250 \times 4^{L}$. For comparison, the Fokker-Planck approximation on the finest discretization level (cf. figure 4.1) is also provided. The left part of the figure corresponds to the evolution of $\tau_{x x}$ for levels $L \in\{0,3,6\}$ (Top: $t \in[0,2]$, Bottom: $t \in[1.4,2]$ ). The right part of the figure describes the evolution of the variances defined by (4.5).

4.2.2. The projection method. The second method is the algorithm by projection, which is based directly on the reflected SDE (1.4). The approximated solution $X_{n+1}^{\Delta t}$ is calculated on each time step (assuming $X_{n}^{\Delta t}$ to be known) via the formula

$$
X_{n+1}^{\Delta t}=\pi\left(X_{n}^{\Delta t}+\left(\kappa\left(t_{n}\right) X_{n}^{\Delta t}-\frac{1}{2 \lambda} X_{n}^{\Delta t}\right) \Delta t+\sqrt{\frac{1}{\lambda}}\left(B_{t_{n+1}}-B_{t_{n}}\right)\right),
$$

where $\pi$ again denotes the projection on $\overline{B(0, R)}$. This scheme is proven in [4] to be weakly convergent of order $\sqrt{\Delta t}$.

The extra-stress tensor can be approximated using expression (2.7). Hence, the direct approximation of $\tau\left(t_{n}\right)$ would be

$$
\begin{aligned}
\tau_{n}^{\Delta t}=- & \frac{\eta_{p}}{\Delta t} \mathbb{E}\left(X_{n+1}^{\Delta t} \otimes X_{n+1}^{\Delta t}-X_{n}^{\Delta t} \otimes X_{n}^{\Delta t}\right) \\
& +\eta_{p} \mathbb{E}\left(\kappa\left(t_{n}\right) X_{n}^{\Delta t} \otimes X_{n}^{\Delta t}+X_{n}^{\Delta t} \otimes X_{n}^{\Delta t} \kappa^{T}\left(t_{n}\right)\right) .
\end{aligned}
$$

The evolution of the $x x$ component of the extra stress tensor $\tau$ along with its variance (4.5) are provided in figure 4.3. The convergence to the Fokker-Planck solution is clearly observed. Not however, that the projection method produced much more noisy solutions than the penalization one. Indeed, the variance in figure 4.3 as about 10 times larger than in figure 4.2

4.2.3. The symmetric Reflexion method. The third method (proposed in [3]) is a slight modification of the previous one and has the advantage of converging weakly with the order of $\Delta t$ rather than $\sqrt{\Delta t}$. The idea is to replace the projection on the ball by the mirror reflection over its boundary. We will refer to it as to the algorithm by symmetric reflection. Each time step of this algorithm is thus composed of the two following sub- steps:

- First update the stochastic process without taking into account the reflecting force 

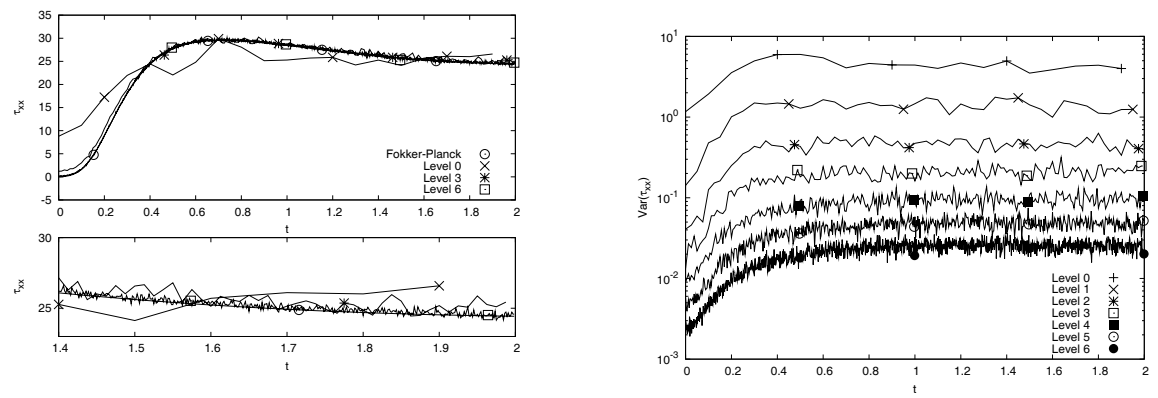

FIG. 4.3. The projection method in shear flow with $\dot{\gamma}=10$ with the same discretization parameters as in figure 4.2 compared to the Fokker-Planck approximation on the finest discretization level (see figure 4.1). The left part of the figure corresponds to the evolution of $\tau_{x x}$ for levels $L \in\{0,3,6\}$ (Top: $t \in[0,2]$, Bottom: $t \in[1.4,2])$. The right part of the figure describes the evolution of the variances defined by (4.5).
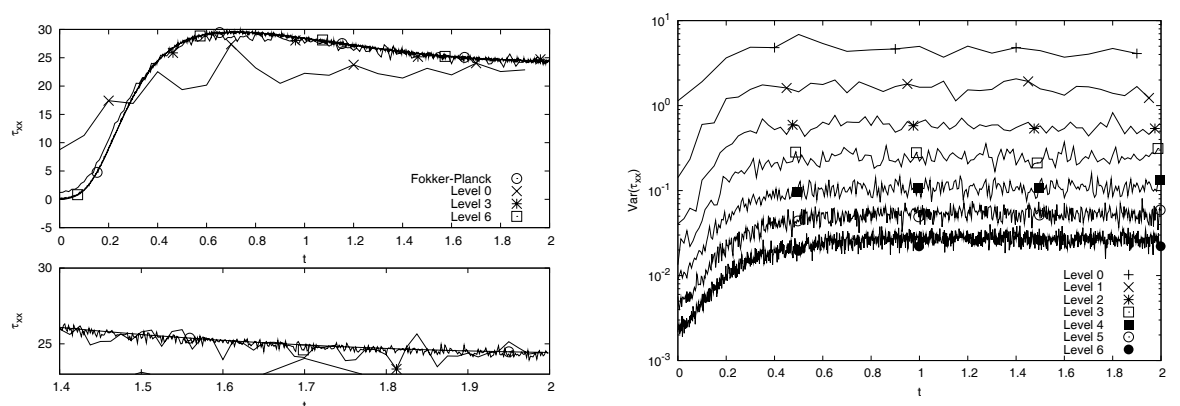

FIG. 4.4. The symmetric reflexion method in shear flow with $\dot{\gamma}=10$ with the same discretization parameters as in figure 4.2 compared to the Fokker-Planck approximation on the finest discretization level (see figure 4.1). The left part of the figure corresponds to the evolution of $\tau_{x x}$ for levels $L \in\{0,3,6\}$ (Top: $t \in[0,2]$, Bottom: $t \in[1.4,2]$ ). The right part of the figure describes the evolution of the variances defined by (4.5).

at the boundary $\partial B(0, R)$, i.e., compute $Y_{n+1}^{\Delta t}$ by

$$
Y_{n+1}^{\Delta t}-X_{n}^{\Delta t}=\left(\kappa\left(t_{n}\right) X_{n}^{\Delta t}-\frac{1}{2 \lambda} X_{n}^{\Delta t}\right) \Delta t+\sqrt{\frac{1}{\lambda}}\left(B_{t_{n+1}}-B_{t_{n}}\right) .
$$

- If no reflection occurs, i.e., $\left|Y_{n+1}^{\Delta t}\right| \leq R$, we set $X_{n+1}^{\Delta t}=Y_{n+1}^{\Delta t}$. Otherwise, we set $X_{n+1}^{\Delta t}$ to be the mirror image of $Y_{n+1}^{\Delta t}$ with respect to $\partial B(0, R)$, i.e.,

$$
X_{n+1}^{\Delta t}=\frac{2 R-\left|Y_{n+1}^{\Delta t}\right|}{\left|Y_{n+1}^{\Delta t}\right|} Y_{n+1}^{\Delta t}
$$

These two cases can be combined into the single formula

$$
X_{n+1}^{\Delta t}=Y_{n+1}^{\Delta t}-2 \frac{\left|Y_{n+1}^{\Delta t}\right|-R}{\left|Y_{n+1}^{\Delta t}\right|} Y_{n+1}^{\Delta t} 1_{\left|Y_{n+1}^{\Delta t}\right|>R}
$$




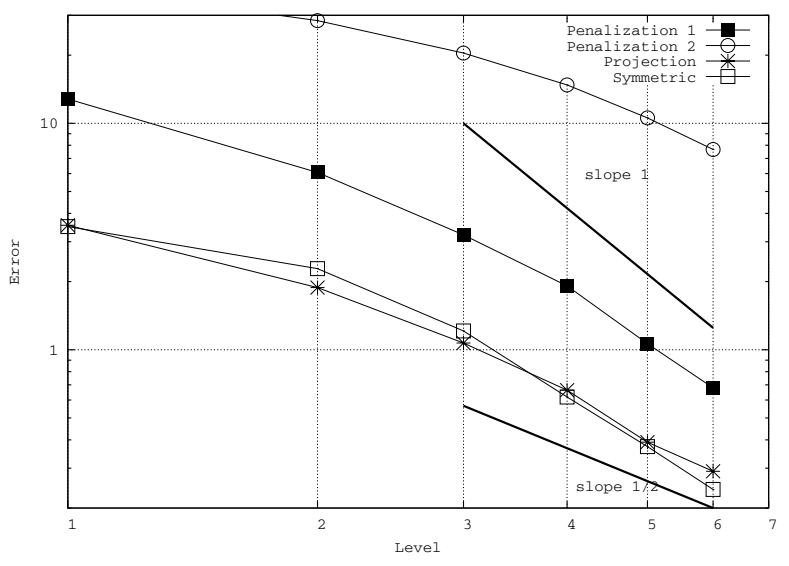

FIG. 4.5. Log-plot of the $L^{2}$-error using the Fokker-Planck simulation $\left(N_{R}=26, N_{F}=16\right.$, $\Delta t=0.1 \times 2^{-6}$ ) as reference solution. Seven different discretization levels $L \in\{0,1,2,3,4,5,6\}$ are considered. For each level $L$, the corresponding numerical parameters are $\Delta t_{L}=0.1 \times 2^{-L}$ and $M_{L}=250 \times 4^{L}$. The "Penalization 1" method corresponds to the choice $\varepsilon=\Delta t$ while "Penalization 2" corresponds to $\varepsilon=\sqrt{\Delta t}$. Slope 1 indicates an order of converge of $O(\Delta t)$ and slope $1 / 2$ indicates an order of converge of $O(\sqrt{\Delta t})$. Only the Penalization method 1 and the symmetric reflexion method exhibit an optimal order of convergence $O(\Delta t)$.

The extra-stress tensor can be approximated as in the projection method using (4.7). The evolution of the $x x$ component of the extra stress tensor $\tau$ along with its variance (4.5) are provided in figure 4.4. The convergence to the Fokker-Planck solution is again observed. The stochastic noise of this method is about the same as of the projection one, cf. figure 4.3.

4.3. Comparison of numerical schemes. We study in figure 4.5 the convergence of the three stochastic methods described above toward the reference FokkerPlanck solution of Subsection 4.1 with respect to the refinement in time. The error is measured in the $L^{2}$-norm in time, namely $\|\tau\|_{0}:=\sqrt{\int_{0}^{T}\left(\tau_{x x}^{2}+\tau_{x y}^{2}+\tau_{y y}^{2}\right)}$. On one hand, it turns out that penalization method with $\varepsilon=\Delta t$ and the symmetric reflexion method demonstrate an optimal order of convergence of $O(\Delta t)$ (slope 1 in figure 4.5). On the other hand, the penalization method with $\varepsilon=\sqrt{\Delta t}$ and the projection method suffer a loss of convergence order and seem to exhibit an order of $O(\sqrt{\Delta t})$ (slope 1/2 in figure 4.5). We also note that according to evolution of the variances reported in figures 4.2-4.4, the penalization method exhibits significantly less noise than the two other methods in the approximation of the stress tensor.

Acknowledgments. $\mathrm{AB}$ and $\mathrm{AL}$ are very grateful to Philippe Clément and Marco Picasso for the help they have provided and the permanent interest they have shown for this research. AL is indebted to Jacques Rappaz for the invitation to the Institute of Analysis and Scientific Computing (IACS), EPFL, which has permitted to finish this work, and to Pingwen Zhang and Claude Le Bris for organizing the workshop on complex fluids in Beijing where these results were presented. 


\section{REFERENCES}

[1] A. Bensoussan and J.L. Lions, Impulse Control and Quasivariational Inequalities, $\mu$. GauthierVillars, Montrouge, 1984. Translated from the French by J.M. Cole.

[2] R. Bird, C. Curtiss, R. Armstrong and O. Hassager, Dynamics of Polymeric Liquids, John Wiley \& Sons, New-York, 1-2, 1987.

[3] M. Bossy, E. Gobet and D. Talay, A symmetrized Euler scheme for an efficient approximation of reflected diffusions, J. Appl. Probab., 41(3), 877-889, 2004.

[4] C. Costantini, B. Pacchiarotti and F. Sartoretto, Numerical approximation for functionals of reflecting diffusion processes, SIAM J. Appl. Math., 58(1), 73-102, (electronic), 1998.

[5] F.G Diaz, J.G Delatorre and J.J Freire, Viscoelastic properties of simple flexible and semirigid models from brownian dynamics simulation, Macromolecules, 23, 3144-3149, 1990.

[6] G.K. Fraenkel, Visco-elastic effect in solutions of simple particles, J. Chem. Phys., 20(4), 642$647,1952$.

[7] B. Jourdain and T. Lelièvre, Mathematical analysis of a stochastic differential equation arising in the micro-macro modelling of polymeric fluids, Probabilistic Methods in Fluids, World Sci. Publishing, River Edge, NJ, 205-223, 2003.

[8] I. Karatzas and S.E. Shreve, Brownian Motion and Stochastic Calculus, Graduate Texts in Mathematics, Springer-Verlag, New York, 113, 1991.

[9] J.L. Menaldi, Stochastic variational inequality for reflected diffusion, Indiana Univ. Math. J., 32(5), 733-744, 1983.

[10] H.C. Öttinger, Stochastic Processes in Polymeric Fluids, Springer-Verlag, Berlin, 1996.

[11] R.G. Owens, A new microstructure-based constitutive model for human blood, J. NonNewtonian Fluid Mech., 140, 57-70, 2006.

[12] R. Pettersson, Penalization schemes for reflecting stochastic differential equations, Bernoulli, 3(4),403-414, 1997.

[13] L.R.G. Treloar, The Physics of Rubber Elasticity, Clarendon Press, Oxford, 1975.

[14] H.R. Warner, Kinetic theory and rheology of dilute suspensions of finitely extendible dumbbells, Ind. Eng. Chem. Fund, 11, 379-387, 1972. 